Top quark theory and the new physics searches frontier

This content has been downloaded from IOPscience. Please scroll down to see the full text. 2013 Phys. Scr. 2013014008

(http://iopscience.iop.org/1402-4896/2013/T158/014008)

View the table of contents for this issue, or go to the journal homepage for more

Download details:

IP Address: 137.138.125.164

This content was downloaded on 19/05/2014 at 13:03

Please note that terms and conditions apply. 


\title{
Top quark theory and the new physics searches frontier
}

\author{
Gilad Perez \\ ${ }^{1}$ CERN Physics Department, Theory Division, CH-1211 Geneva 23, Switzerland \\ ${ }^{2}$ Department of Particle Physics and Astrophysics, Weizmann Institute of Science, Rehovot 76100, Israel \\ E-mail: jasgilperez@gmail.com
}

Received 1 October 2013

Accepted for publication 13 November 2013

Published 6 December 2013

Online at stacks.iop.org/PhysScr/T158/014008

\begin{abstract}
We review the special role that the top sector is playing in the context of electroweak (EW) symmetry breaking within and beyond the Standard Model. We briefly report on the current status of the Large Hadron Collider (LHC) 'battle of naturalness' related to top partner searches. The notion of 'mini' intensity and energy frontiers at the LHC is then explained with an example given for each of these fronts. The phase diagram of the Standard Model obtained by extrapolating the relevant basic parameters, in particular the top-Higgs Yukawa coupling to high scales is also mentioned. We finally address some interesting aspects of top physics that are not related to EW physics. In particular, the impressive progress made recently on the next-to-next-to leading order evaluation of the top pair production cross-section is described and the status of the forward-backward top asymmetry is also summarized.
\end{abstract}

PACS number: 14.65.Ha

(Some figures may appear in colour only in the online journal)

\section{Introduction}

The top quark is a rather unusual creature, especially when compared with the other known elementary fermions. The top is the most massive point-like object known to exist, with a mass of around $173 \mathrm{GeV}$ [1]. Within the Standard Model (SM) this large mass is translated to a sizable Yukawa coupling to the, recently discovered, Higgs boson, $y_{t}\left(m_{t}\right)$ $\simeq 1$. In the quantum world this coupling implies perturbative interactions, in the sense that radiative processes involving the top-Higgs Yukawa couplings are typically suppressed by $\mathcal{O}\left(2 N_{c} y_{t}^{2} / 16 \pi^{2}\right)=4 \%$, a pretty small number. Yet apart from the Cabibbo-Kobayashi-Maskawa (CKM) charge parity (CP) violating phase all the other parameters that characterize the SM fermion sector are much smaller and hierarchical. The mass of the next to heaviest fermion, the bottom quark, is roughly $3 \mathrm{GeV}$ (at the $Z$ mass see [2] for details), and its corresponding Yukawa coupling is less than 2\%. (In some sense, therefore, the top quark is the only known 'normal' fermion with order one gauge and Yukawa interactions strength, etc.) Furthermore, the top width is larger than $\Lambda_{\mathrm{QCD}}$ so that it decays before hadronizing. The top width is large but not too large such that $\Gamma_{\mathrm{t}} \ll m_{\mathrm{t}}$. Consequently, it allows one to use a narrow width approximation when describing its decay process. Then despite the fact that the top is a colored object the distribution of its daughter product can be captured quite nicely by using leading order computations. The distributions of the top daughter products makes it possible to access its spin/helicity configuration. The same holds for the top charge that can be probed thought the charge of the outgoing hard lepton in semi-leptonic (SL) decays. In addition, it opens a window for a new way of doing flavor precision tests, as the flavor of the outgoing bottom at the time of decay is also effectively tagged by the lepton charge [3]. The top large mass and width makes its physics perturbative and thus leads to a precision program that started in the Tevatron era (see [4] for a recent review) and is being pursued with full strength currently at the LHC (see e.g. [1]). Finally, there are two important aspects of top physics associated with Higgs physics. Both are related to the way the top quark impact Higgs and electroweak (EW) symmetry breaking at the quantum level. The fact that the top has a significant coupling to the Higgs leads to two potentially profound consequences. The first is that the Higgs potential, in particular its quartic coupling is driven to negative values due to its coupling to the top. As a consequence if extrapolated up to high energies our current EW vacuum might be metastable [5]. The second is that it seems that radiative corrections involving a virtual top 
pair lead to a severe fine tuning where the Higgs mass square is sensitive to contributions at the microscopic cutoff of the theory.

\section{Top and EW physics}

The discovery of the Higgs boson at the Large Hadron Collider (LHC) is a great victory for the SM. With its minimal scalar sector of EW breaking, the SM at short distances, well below the proton radius, is a complete weakly coupled theory. Even though the SM cannot explain some experimental observations such as neutrino masses, the baryon asymmetry of the universe and the origin of dark matter, one cannot deduce with any certainty an energy scale for which the SM would be forced to be extended (with the exceptions of the Planck scale related to gravity, and the fantastic ultra-high scale of the Landau pole of the $U(1)$ hyper charge force). However, as mentioned, the fact that the Higgs mass is subject to additive renormalization implies that the EW scale is unnatural. The solution of this UV sensitivity problem requires new dynamics characterized by an energy scale close to the weak scale ${ }^{3}$. As already mentioned, the most severe fine tuning problem arises from quantum processes involving the Higgs-top coupling. A simple possibility to stabilize the EW scale in a controlled manner is to add some new particles with the same gauge quantum numbers as the top ones. The stability is obtained when the virtual contributions of the new particle exactly cancel the ones coming from the SM tops. These new states are collectively denoted as top partners. In known examples the partners might be scalars as in the celebrated case of supersymmetry (SUSY) or fermions as in the case of composite Higgs models (CHMs). Current bounds on the top partner masses is of order of the 700 (800) for supersymmetric scalar (composite-Higgs fermionic) states (see e.g. the talks by R Chierici and A Parker given in this meeting).

While these bounds are fairly strong they are not bullet proof and also they result only in a moderate naturalness pressure (we shall not dwell here on a discussion on the various definitions of tuning). Probably, the most relevant question amidst the 'LHC battle for naturalness' is how are we going to discover or improve the bounds on the top partners both in terms of (i) mass reach; and in terms of (ii) robustness. These two criteria can possibly direct us to where the theoretical, phenomenological and experimental effort is made. One can define two 'mini-frontiers' for the battle for naturalness at the LHC.

(i) The mini-energy frontier, where the effort is focused on searching for ultra-massive partners, which is dominated

\footnotetext{
3 Giving up on this solution implies accepting a 'desert-like' paradigm, namely forbidding the Higgs, and the tops that couple quite strongly to the Higgs, to significantly couple to any form of dynamics that exists down to imaginary microscopic scales many of orders of magnitude below the ones that we currently directly probed (see $[6,7]$ for recent discussions). This approach sounds a bit like the state of mind of the physics community toward the end of the 19th century, where the common thinking was that apart from some small puzzles the program of understanding the basic laws of nature is close to completion. Another alternative approach argues that, with analogy to the explanation for the smallness of the cosmological constant [8], the weak scale is determined through the environmental selection principle [9]. However, this explanation seems to be less robust $[10,11]$.
}

by the highest center of mass energies that can be reached by the LHC.

(ii) The mini-intensity frontier, where the effort is focused on searching for partners with mass below or near the current bounds, however, when the partners are elusive, namely, where for some reason the current searches are not sensitive enough to their presence.

The physics describing these two frontiers is qualitatively different both in terms of the phenomenology describing them and in terms of the necessary experimental corresponding effort. We shall now briefly discuss some aspects of these two frontiers.

\subsection{The LHC mini-energy frontier}

As the LHC bounds continue to improve, the top partner masses are expected to be pushed above the TeV scale, and the gluino or $t \bar{t}$ resonance masses could be pushed toward the $3 \mathrm{TeV}$ scale. One would then be entering the boosted phenomenology regime as follows. Consider, for instance, searches for heavy $t \bar{t}$ resonances. Due to their large mass their decay products carry very large momenta. Consequently, the top daughter particles collimate and get clustered into a single jet $[12,13]$. These precious events, with highly boosted tops, look similar to mundane quantum chromodynamics (QCD) two-jet processes and thus might be swamped by the background. In recent years the community has developed several theoretical and experimental approaches to deal with this challenge, they all generically deal with the physics of jet substructure or massive boosted jets (see [14] for a recent review).

Two main approaches have emerged: filtering [15] (see also $[16,17])$ and the template overlap method [18]. Filtering algorithms act on the list of jet constituents by removing the soft components based on some measure which defines the 'hard' part of the jet. The remaining constituents are then reclustered into the 'filtered' jet. The template overlap method compares the jet to a set of parton level states built according to a fixed-order distribution of signal jets called templates. The comparison makes use of an 'overlap function' which evaluates the level of agreement between each measured jet and a set of templates. Almeida et al [18] focused on building the templates according to the leading order decay modes, namely two body $(N=2)$ for the boosted Higgs and three body $(N=3)$ for the boosted top.

There have only been a few studies on the ability of jet substructure methods to improve the LHC searches for ultra heavy partners, although a similar challenge would apply to this case, as is illustrated by figures 1 and 2 . In the former we show the decay of a moderate mass top partner, $t^{\prime}$ (into a top and a $W$, see e.g. [19]), while in figure 2 the case where the top partner is heavy resulting with a highly boosted outgoing top and a $W$ that might look similar to the corresponding QCD multi-jet (or EW+jets) background (or one need to settle for leptonic-only final states, with smaller branching ratios, adopting mini-isolation techniques [20]). In [21] the search for intermediate mass stops with the HEPTopTagger was analyzed. In [22] boosted tops were studied via the Johns Hopkins University (JHU) [23] algorithm. In [24] the authors studied the ultra-heavy stops region, as well as 


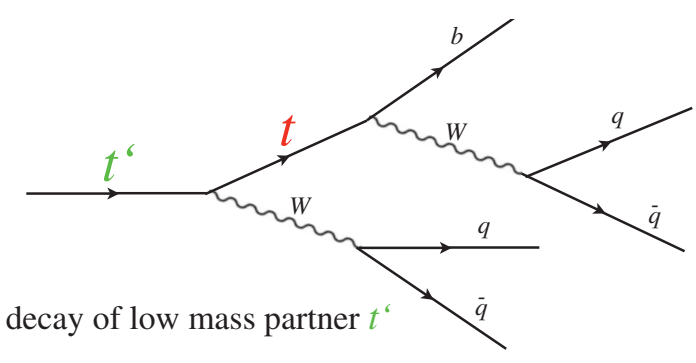

Figure 1. A typical decay of a moderate mass $t^{\prime}$ fermionic partner in CHMs. The outgoing top and $W$ are not very boosted and their decay products can be clustered separately.

their mixing parameters, using an optimized algorithm that combines ingredients from the HEPTopTagger, and the JHU and compact muon solenoid (CMS) [25] top taggers. In [26] a search for boosted tops from gluino decays was presented based on the JHU tagger. All of the above studies use filtering to identify the boosted tops coming from the decays of heavy stops or gluinos.

\subsection{The LHC mini-intensity frontier}

In this part we discuss the case in which the top partner's mass is below the current experimental bounds, and for some reason they are elusive. Within SUSY, the possibility that the stops are light but evade current searches is certainly not new. To understand how the experimental bounds are obtained it is convenient to organize the searches using the framework of simplified models. The most sensitive searches are based on either the case where the stop decays to a top quark and the lightest SUSY particle (LSP), $\tilde{t} \rightarrow t \chi^{0}$, or the case when it decays to a $b$ quark and a chargino that further decays to a $W$ and the LSP, $\tilde{t} \rightarrow b \chi^{+} \rightarrow b W^{+} \chi^{0}$. Needless to say, these final states are model dependent. However, what is common to both of these cases as well as many other SUSY searches is the presence of an LSP in the final state. This implies that all of these events will be characterized by a large source of missing transverse energy. To suppress the SM backgrounds, the experimentalists therefore typically require that the events contain a large amount of missing energy. Current model building efforts have thus been aimed at minimizing the latter.

There are two main approaches to achieving this: (a) if the SUSY spectrum is compressed, e.g. when the stop and the LSP (or other daughter particles in a stealth scenario) are close in mass, the outgoing LSP tends to be softer, making the missing-energy-based searches inefficient [27-29]; (b) if $R$-parity is not conserved, missing energy is completely absent from signal events [30-35]. Experimental searches for such scenarios are already underway $[36,37]$.

We shall now discuss another mechanism to make the partners more elusive that we shall call flavorful naturalness. The top partners can be defined according to their coupling to the Higgs in order to satisfy their role as EW-scale stabilizers. Naively, therefore, one might conclude that flavor physics and naturalness are completely decoupled. However, even within a minimal top partner sector the question regarding the flavor structure of the model might not be trivial. The top partners need not be mass eigenstate fields in order to yield a sufficient cancelation to the quantum contributions to the Higgs mass, and thus even a single-top-partner model might lead to flavor and $\mathrm{CP}$ violation effects. In fact, this feature was recently exploited in [38]. It was demonstrated that in SUSY the top squark flavor eigenstate can consist of an admixture of would be stop-like and scharm-like mass eigenstates.

The possibility of the top partners being an admixture of several eigenstates raises an important question, namely, what can we say robustly about the flavor structure of the partners. Usually, this question is suppressed because of theoretical prejudices and possible simplistic interpretation of the bounds coming from low energy flavor changing neutral current processes (FCNCs). Most of the studies of naturalness assumed either flavor universality among the partners or an approximate $U(2)$ symmetry which acts on the partner of the first two generations. However, a recent analysis of the constraints coming from $D-\bar{D}$ mixing and $K-\bar{K}$ mixing shows that partner's degeneracy is not required within models of down alignment [39]. Models in which the new physics couplings are diagonal in the mass basis were considered both in the context of SUSY [40, 41] and within the framework of composite Higgs [42, 43].

What makes the possibility of partner's non-degeneracy even more interesting are the following two facts shown to hold for supersymmetric models: (a) the direct experimental bounds on the second generation squarks is rather weak, of the order of $400-500 \mathrm{GeV}$. This is since the searches are mainly sensitive to the 'valence' squark masses and are optimized to look for heavy squarks [44]. (b) As mentioned, if the top partners are not pure mass eigenstates but rather form an admixture of, for example within supersymmetry, stop-like and scharm-like squarks, the way the direct searches are performed needs to be modified. The final states relevant to this search would not only involve top-pairs (and missing energy) but also charm-pairs and top-charm final states resulting in weaker bound on the stop mass [38]. Combining (a) and (b) leads to supersymmetric 'flavorful naturalness' where despite the non-trivial flavor structure of the top sector, the level of fine tuning is similar to the conventional pure stop mass eigenstate scenario or even improved.

\subsection{Top and EW stability}

Despite what was said above about naturalness and how it provides a motivation for the presence of $\mathrm{TeV}$ new physics, the fact is that as of today no non-SM state has been observed. Thus, our only option currently is to use whatever new information, direct or indirect, brought to us by the LHC to investigate whether we get any indications for new physics. In this respect, the Higgs potential is a particularly rich source of information:

$$
V=\text { const. }+m_{\mathrm{H}}^{2}|H|^{2}+\lambda|H|^{4} .
$$

The measured values of all three parameters in equation (1) raise theoretical concerns and turn out to be close to 'living dangerously' conditions. The constant in equation (1) (which actually represents the full vacuum energy of the theory) leads to the cosmological constant problem and $m_{\mathrm{H}}^{2}$ embodies the problem of Higgs naturalness. The recent measurement of the Higgs boson mass has added a new ingredient to the list; $\lambda$ is special with respect to vacuum stability [5]. Small 


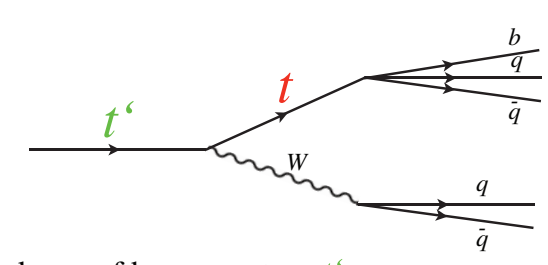

decay of heavy partner $t$

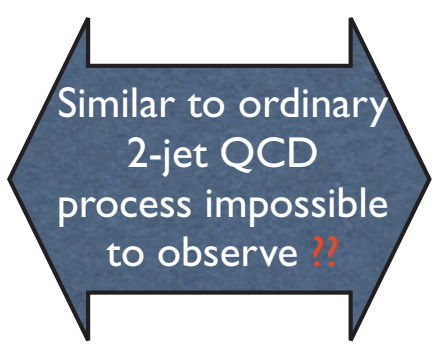

Figure 2. Decay of ultra-high mass $t^{\prime}$ fermionic partner in CHMs. The outgoing top and $W$ are highly boosted and their decay products collimate. Techniques involving jet substructure (and mini-leptonic isolation) are required to separate the signal from the much larger QCD (and EW+jets) background.

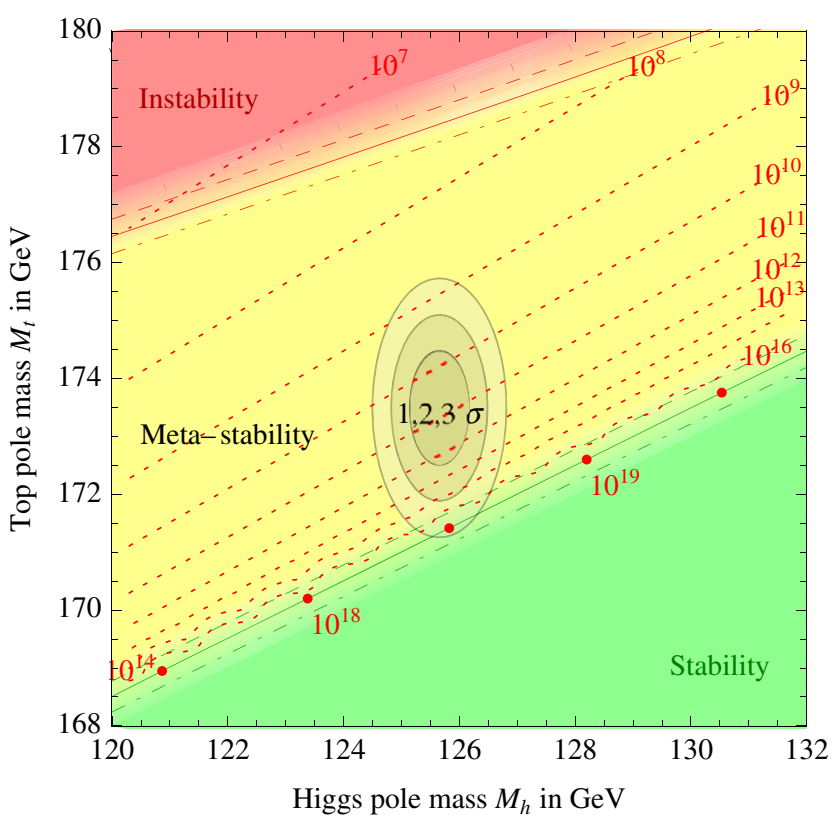

Figure 3. Zoom in of the region of the preferred experimental range of the Higgs and top mass (the gray areas denote the allowed region at 1,2 and $3 \sigma$ 's), the dotted contour-lines show the instability scale in $\mathrm{GeV}$ (taken from [5]).

variations of any of these three parameters with respect to their measured values could have devastating consequences for our life-friendly universe. The situation for $\lambda$ is quantified in figure 3 where the phase diagram of the SM EW scale is shown. The regions are shown for the top and Higgs masses, which within the SM are directly related to the top Yukawa and Higgs quartic coupling respectively (for details about this figure, see [5]). If the SM is assumed to be valid up to very short distances (much beyond what can be tested experimentally today but below the Planck scale), the measured Higgs boson mass corresponds to a near-critical situation in which the EW vacuum is on the verge of instability.

\section{More top physics}

\subsection{The next-to-next-to-leading (NNLO) frontier}

Regardless of beyond the SM physics, the top quark pair production is one of the cornerstones of the SM program at hadron colliders, and a number of precision calculations of this process have appeared in the recent past. A major progress was made in the last year or so where the
Table 1. Unofficial summary of $t \bar{t}$ production cross-sections, summary taken from [49].

\begin{tabular}{llll}
\hline Collider & $\sigma_{\text {tot }}(\mathrm{pb})$ & Scales $(\mathrm{pb})$ & PDF $(\mathrm{pb})$ \\
\hline Tevatron & 7.164 & $+0.110(1.5 \%)$ & $+0.169(2.4 \%)$ \\
& & $-0.200(2.8 \%)$ & $-0.122(1.7 \%)$ \\
LHC 7 TeV & \multirow{2}{*}{172.0} & $+4.4(2.6 \%)$ & $+4.7(2.7 \%)$ \\
& & $-5.8(3.4 \%)$ & $-4.8(2.8 \%)$ \\
LHC 8 TeV & \multirow{2}{*}{245.8} & $+6.2(2.5 \%)$ & $+6.2(2.5 \%)$ \\
& & $-8.4(3.4 \%)$ & $-6.4(2.6 \%)$ \\
LHC 14 TeV & \multirow{2}{*}{953.6} & $+22.7(2.4 \%)$ & $+16.2(1.7 \%)$ \\
& & $-33.9(3.6 \%)$ & $-17.8(1.9 \%)$ \\
\hline
\end{tabular}

total inclusive cross-section became known in full to the next-to-next-to-leading order (NNLO) accuracy [45-48]. In table 1 we present an unofficial compilation of the various relevant $t \bar{t}$ production cross-sections with the corresponding uncertainties (taken from [49]).

Given such a level of accuracy the first question that one may ask is whether the SM predictions are in agreement with the rather precise measurements made at the Tevatron and the LHC experiments. To keep the discussion simple let us consider for instance the fixed order predictions, not including soft gluon resummation. In [50] one can find the LO, NLO and NNLO results compared with the actual data. Each result is computed with a PDF set of matching accuracy (using the MSTW2008 (68cl) family of PDF sets [51]). Similar results are obtained if other PDF sets such as CT10 [52] and NNPDF2.3 [53] are used; see [54] for a detailed comparison of the predictions from the various sets.

In figure 4 (left) the authors of [50] show the scale dependence of the predicted cross-section at the Tevatron, as a function of the top quark mass. We note the significant and consistent improvement in the theoretical precision due to the inclusion of corrections at higher perturbative orders. The full theoretical uncertainty is, roughly, about twice as large as the scale dependence one (from parton distribution functions, etc). The latest Tevatron measurement with its error is also shown on the plot. Next we turn to the LHC. In figure 4 (right) we show the scale dependence of the predicted cross-section at the LHC $8 \mathrm{TeV}$ as a function of $m_{\text {top }}$. Similarly to the case of the Tevatron, a very good perturbative convergence of the theoretical prediction and good agreement with the available measurement is obtained (see [1] for a experimental summery regarding the relevant measurements). 

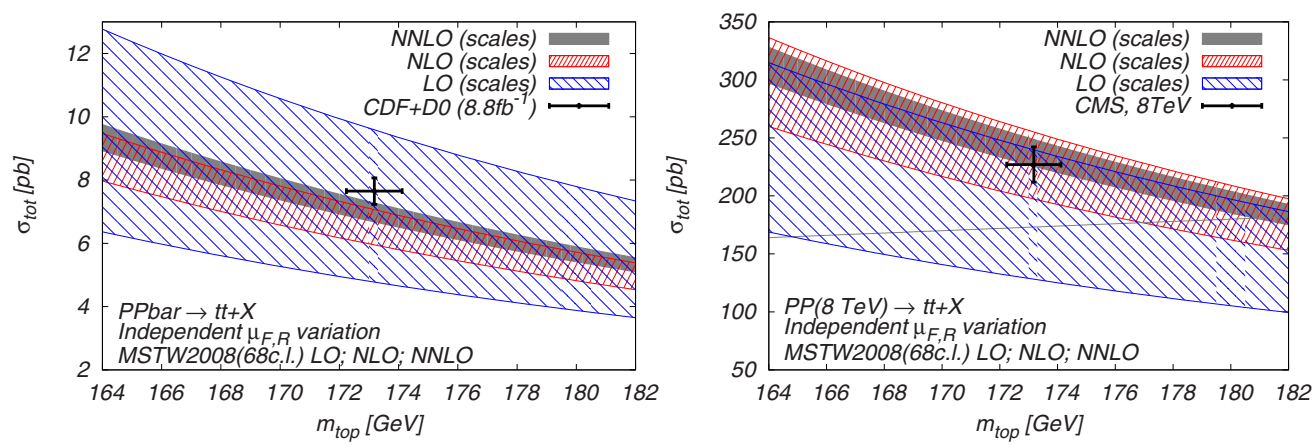

Figure 4. Scale dependence of the total cross-section at LO (blue), NLO (red) and NNLO (black) as a function of $m_{\text {top }}$ at the Tevatron (left) and the LHC $8 \mathrm{TeV}$ (right). No soft gluon resummation is included. For reference the most precise experimental measurements are also shown. Figure is taken from [50].

The above dramatic progress in our theoretical knowledge of the top production cross-section can be used as an important consistency check for other methods to extract the top mass (see [55] for a recent discussion), however, at present it cannot compete with the incredible experimental accuracy already reached by other dedicated methods [56]. Clearly, an improved determination of the top mass would also have important implications for our sensitivity to new physics models as well as to improve the precision of our extrapolation of the SM EW phase diagram, mentioned above.

So far the progress achieved in the NNLO frontier has been mostly related to the inclusive production cross-section. There are various open questions to be addressed that require an understanding of the differential distribution of the $t \bar{t}$ cross-section. Two very important examples are the top-pair spectrum or invariant mass distribution at large values, and the $t \bar{t}$ forward-backward asymmetry that is discussed in section 3.2 .

\subsection{The $t \bar{t}$ forward-backward asymmetry}

Within the SM, the $t \bar{t}$ forward-backward asymmetry, $A_{t \bar{t}}$, is an interesting variable because it tells us about QCD interactions beyond the leading order but in a region that should be well described by the perturbation theory $[57,58]$. Furthermore, as the SM contributions are expected to be small [57-61], the measurement of $A_{t \bar{t}}$ is sensitive to beyond-the-SM contributions. The asymmetry is quite a special observable since shifting it requires new physics with non-standard couplings both to the $t \bar{t}$ quark current as well as to the current of $u \bar{u}$ (or possibly $d \bar{d}$ ) initial-state quarks.

Both Tevatron experiments, CDF and DØ, have observed an anomalously large forward-backward asymmetry in $t \bar{t}$ production, defined by

$$
A_{t \bar{t}}=\frac{N\left(\Delta y^{t \bar{t}}>0\right)-N\left(\Delta y^{t \bar{t}}<0\right)}{N\left(\Delta y^{t \bar{t}}>0\right)+N\left(\Delta y^{t \bar{t}}<0\right)},
$$

where $\Delta y^{t \bar{t}} \equiv y_{t}-y_{\bar{t}}\left(y_{t}\right.$ being the top rapidity) and $N$ is the total number of events satisfying the corresponding constraint. This asymmetry has been measured in SL decays with the

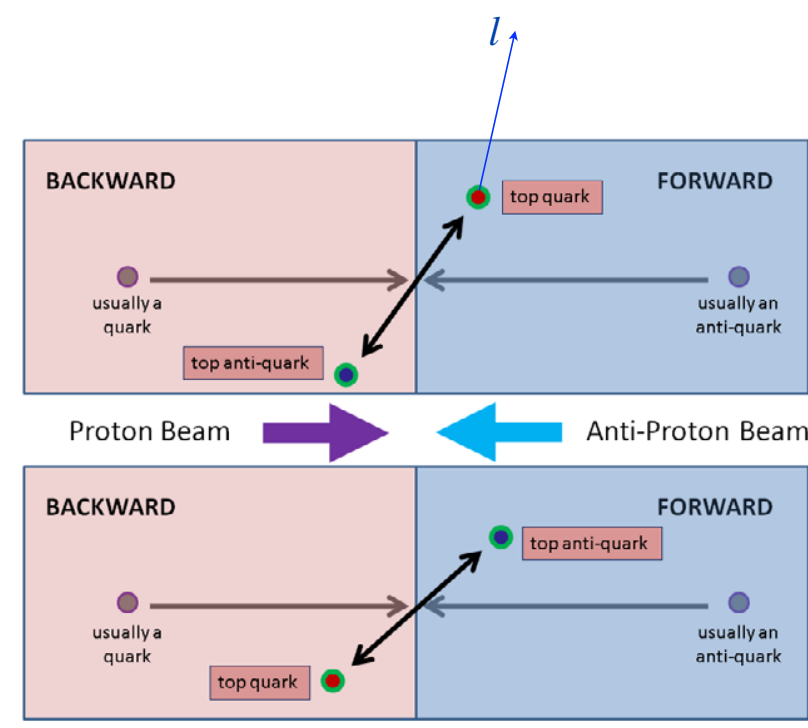

Figure 5. The two types of forward-backward asymmetries associated with top physics: the $t \bar{t}$ partonic (lepton-based) asymmetry is illustrated in the lower (upper) panel.

following result:

$$
\begin{aligned}
& A_{t \bar{t}}(\mathrm{CDF})=0.164 \pm 0.047, \quad[62], \\
& A_{t \bar{t}}(\mathrm{D} \varnothing)=0.196 \pm 0.065, \quad[63],
\end{aligned}
$$

to be compared with the SM NLO prediction with EW corrections included [64]

$$
A_{t \bar{t}}(\mathrm{SM})=0.088 \pm 0.006
$$

Although not statistically significant for a discovery, the observed excess is consistent among experiments. Moreover, the excess in the top asymmetry is accompanied by several excesses in lepton-based asymmetries measured at the Tevatron in the SL and di-leptonic (DL) channels. The two types of independent forward-backward asymmetries associated with top physics are illustrated in figure 5 .

The current results for inclusive lepton-based asymmetries together with the SM prediction (as reported by 
the experimental collaborations) are

$$
\begin{array}{ll}
\text { SL }: & A_{\ell}(\mathrm{CDF})=0.094_{-0.029}^{+0.032}, \\
& A_{\ell}(\mathrm{SM})=0.038 \pm 0.003, \quad[65], \\
\mathrm{SL}: & A_{\ell}(\mathrm{D} \varnothing)=0.047 \pm 0.023_{-0.014}^{+0.011}, \\
& A_{\ell}(\mathrm{SM})=0.023, \quad[66], \\
\mathrm{DL}: & A_{\ell}(\mathrm{D} \varnothing)=0.044 \pm 0.037 \pm 0.011, \\
& A_{\ell}(\mathrm{SM})=0.038 \pm 0.003, \quad[67], \\
\mathrm{DL}: & A_{l l}(\mathrm{D} \varnothing)=0.072 \pm 0.060, \\
& A_{l l}(\mathrm{SM})=0.038 \pm 0.003, \quad[68], \\
\mathrm{DL}: & A_{l l}(\mathrm{CDF})=0.123 \pm 0.054 \pm 0.015, \\
& A_{l l}(\mathrm{SM})=0.048 \pm 0.004, \quad[67],
\end{array}
$$

where the single and double-lepton based asymmetries are defined as follows

$$
A_{\ell}=\frac{N(q \times \eta>0)-N(q \times \eta<0)}{N(q \times \eta>0)+N(q \times \eta<0)}
$$

and

$$
A_{l l}=\frac{N(\Delta \eta>0)-N(\Delta \eta<0)}{N(\Delta \eta>0)-N(\Delta \eta<0)}
$$

with $q$ and $\eta$ the charge and pseudorapidity of the lepton and $\Delta \eta \equiv \eta_{l^{+}}-\eta_{l^{-}}$.

A puzzling aspect of the observed excess is that the large value of the measured asymmetries are not accompanied by any sizeable deviation in other top observables, like the total or differential $t \bar{t}$ production cross-sections. This strongly constrains possible explanations of the anomalous forward-backward asymmetry. The main obstacle for a satisfactory understanding of this anomaly is the fact that the Tevatron ceased its operations in 2011. With all—or most of - the data already analyzed new insight on the asymmetry can only come from a smart choice of new observables to investigate with the Tevatron data or from the LHC data.

Being a proton-proton collider, the LHC is not sensitive to $A_{t \bar{t}}$. However, the LHC can probe a related observable-the charge asymmetry in $t \bar{t}$ production, $A_{C}$. Measurements of $A_{C}$ at $\sqrt{s}=7 \mathrm{TeV}$ have been reported by both ATLAS [69] (for $4.7 \mathrm{fb}^{-1}$ ) and CMS [70, 71] (for $5.0 \mathrm{fb}^{-1}$ ). The expected SM asymmetry $[57,58,64], A_{C}^{\mathrm{SM}}=0.0115 \pm 0.0006$, is much smaller than the Tevatron's asymmetries, due to the domination of the gluon-gluon production channel, which is symmetric. The LHC measurements so far are consistent with the SM value, but the size of the statistical and systematic uncertainties is such that one cannot yet exclude the consistency with the anomalous Tevatron $A_{t \bar{t}}$ result. It is important to emphasize that even within the SM the Tevatron and LHC observables differ in nature. In particular, the dominant $t \bar{t}$ production mechanism and the kinematical reaches available to the top quarks are clearly very different at the two colliders; the Tevatron collides charge-asymmetric beams and top quark production is dominated by quark-antiquark annihilation, while, at the LHC, collisions are charge symmetric and the top pair production is driven by gluon-gluon collisions. Furthermore, non-SM dynamics can naturally induce a large deviation for the forward-backward asymmetry at the Tevatron without affecting the charge asymmetry at the LHC [72-75].
Given that the LHC probes a different observable, we turn our attention back to the Tevatron. The discrepancy between the SM predictions and the measured asymmetries at the Tevatron could be due to an unknown QCD effect, or an unidentified experimental bias. Alternatively, it might be a hint of dynamics beyond the SM (for a review, see e.g. [76]). Either way, the current situation is not satisfying, and an important goal is to investigate what other information can be used to gain more insight.

A step toward getting the most out of Tevatron data was made in [77]. In that article, it was shown that the study of the correlation of $A_{t \bar{t}}$ with a lepton-based asymmetry $A_{\ell}$, measured as a function of some kinematical variable, like the lepton $p_{T}$ or the $t \bar{t}$ invariant mass, can be a powerful discriminating observable. The basic idea behind this proposal is simple, at least in principle. In the SM, the lepton-based asymmetry in $t \bar{t}$ events is completely determined by the $t \bar{t}$ asymmetry, meaning for a given $A_{t \bar{t}}$ one can use top quark decay kinematics to predict $A_{\ell}$. Radiation originating from the top quark decay products alters the kinematics and blurs the relationship between $A_{\ell}$ and $A_{t \bar{t}}$, however this effect is suppressed by the narrow width of the top quark. This relationship is true for the inclusive asymmetries, but also differentially - taking the asymmetries with respect to a kinematic variable $x$; in each bin of $x$, the lepton asymmetry can be fixed knowing $A_{t \bar{t}}$ in that bin, such that $A_{\ell}(x)\left[A_{t \bar{t}}(x)\right]$ traces a calculable curve as $x$ is varied.

However, once we move beyond the SM, $A_{\ell}$ and $A_{t \bar{t}}$ are generically independent. At high $m_{t \bar{t}}, A_{\ell}$ is indeed driven by the top quark kinematics and polarization [12, 78-83], however, near the $t \bar{t}$ threshold $A_{\ell}$ is set by the initial-state quark polarization rather than anything related to the top quarks [84]. Thus, given some observable $x$ that interpolates between the threshold and high- $m_{t \bar{t}}$ regions (lepton $p_{\mathrm{T}}, H_{\mathrm{T}}$, etc), the curve $A_{\ell}(x)$ versus $A_{t \bar{t}}(x)$ will be different for models beyond the SM. Our proposal is to use $x=p_{\mathrm{T}}^{l}$ (the lepton $p_{\mathrm{T}}$ ) and to simultaneously measure $A_{l}\left(p_{\mathrm{T}}^{l}\right)$ and $A_{t \bar{t}}\left(p_{\mathrm{T}}^{l}\right)$, to verify whether the curve $A_{l}\left(p_{\mathrm{T}}^{l}\right)\left[A_{t \bar{t}}\left(p_{\mathrm{T}}^{l}\right)\right]$ is in agreement with the SM. We choose the lepton $p_{\mathrm{T}}$ as our kinematic variable because it is experimentally clean and easy to reconstruct.

\section{Conclusions}

We have discussed several interesting aspects of top quark physics. The top unique properties make it particularly interesting for the high energy community. From the experimental perspective it is a complex structure with many handles that translates to a very rich set of observables to probe. From the perturbative QCD side the top is an object that enables theorists to make very precise computations that yield accurate predictions to test against data. Within the $\mathrm{SM}$, the top quark is also linked to flavor and EW physics due to its large Yukawa couplings. In fact, despite being perturbative, the sizable top Yukawa couplings imply that the top interactions at the quantum level dominate many of the flavor-violating observables as well as the contributions to various EW observables. These features by themselves provide a fairly good motivation to transform top physics into a generic sensitive tool for new physics searches. However, 
what is really singling out top physics as a major player in the new physics searches frontier is the fine tuning problem. We now know for a fact that the Higgs boson exists, and it happens to be pretty light. We are also reasonably certain that the SM Higgs mechanism, with its fundamental scalar, plays a dominant role in EW symmetry breaking. This implies that the Higgs mass is subject to large quantum corrections. The largest corrections are induced by the top-Higgs couplings. As is well known, the only well established and concrete mechanism to solve this UV sensitivity problem is to extend the top sector to include new light 'top-partners' that would counter balance the top quantum corrections to the Higgs mass. Thus, studying top physics is expected to shed light on the mechanism of EW scale stabilization. It transforms top physics to a window for new physics searches with a rough scale associated to them that is expected to be within the LHC's reach. Finally, if one is feeling bold enough to make yet another speculative step and extrapolate the SM Higgs potential up to ultra-high energies (but below the Planck scale) he learns that the phase diagram of the SM is such that the Higgs-top system lies near the critical region of meta-stability at the boundary of stability and instability [5]. This might be a mere coincidence, and any sign of new physics at the LHC will make this discussion obsolete; however, the current state of affairs leaves the possibility that the fact that the top Yukawa is close to its critical value is a hint of some new principle yet to be understood [85-87].

\section{Acknowledgments}

The work of GP was supported by grants from GIF, IRG, ISF, Minerva and the Gruber award.

\section{References}

[1] Schilling F-P (ATLAS Collaboration, CDF Collaboration, CMS Collaboration, D0 Collaboration) 2013 EPJ Web Conf. 4904001

[2] Xing Z-Z, Zhang H and Zhou S 2008 Phys. Rev. D 77113016

[3] Gedalia O et al 2013 Phys. Rev. Lett. 110232002

[4] Buras A J, Perez G, Schwarz T A and Tait T M 2012 Eur. Phys. J. C 722105

[5] Buttazzo D et al 2013 arXiv:1307.3536

[6] Farina M, Pappadopulo D and Strumia A 2013 J. High Energy Phys. JHEP08(2013)022

[7] Giudice G F 2013 arXiv:1307.7879

[8] Weinberg S 1987 Phys. Rev. Lett. 592607

[9] Agrawal V, Barr S M, Donoghue J F and Seckel D 1998 Phys. Rev. D 575480

[10] Harnik R, Kribs G D and Perez G 2006 Phys. Rev. D 74035006

[11] Gedalia O, Jenkins A and Perez G 2011 Phys. Rev. D 83115020

[12] Agashe K, Belyaev A, Krupovnickas T, Perez G and Virzi J 2008 Phys. Rev. D 77015003

[13] Lillie B, Randall L and Wang L-T 2007 J. High Energy Phys. JHEP09(2007)074

[14] Altheimer A et al 2012 J. Phys. G : Nucl. Part. Phys. 39063001

[15] Butterworth J M, Davison A R, Rubin M and Salam G P 2008 Phys. Rev. Lett. 100242001

[16] Krohn D, Thaler J and Wang L-T 2010 J. High Energy Phys. JHEP02(2010)084

[17] Ellis S D, Vermilion C K and Walsh J R 2010 Phys. Rev. D 81094023
[18] Almeida L G, Lee S J, Perez G, Sterman G and Sung I 2010 Phys. Rev. D 82054034

[19] Contino R and Servant G 2008 J. High Energy Phys. JHEP06(2008)026

[20] Rehermann K and Tweedie B 2011 J. High Energy Phys. JHEP03(2011)059

[21] Plehn T, Spannowsky M, Takeuchi M and Zerwas D 2010 J. High Energy Phys. JHEP10(2010)078

[22] Bandyopadhyay P and Bhattacherjee B 2011 Phys. Rev. D 84035020

[23] Kaplan D E, Rehermann K, Schwartz M D and Tweedie B 2008 Phys. Rev. Lett. 101142001

[24] Bhattacherjee B, Mandal S K and Nojiri M 2012 arXiv: 1211.7261

[25] CMS Collaboration 2009925377 CMS-PAS-JME-09-001

[26] Berger J, Perelstein M, Saelim M and Spray A 2011 arXiv:1111.6594

[27] Alwall J, Le M-P, Lisanti M and Wacker J G 2008 Phys. Lett. B 66634

[28] Fan J, Reece M and Ruderman J T 2011 J. High Energy Phys. JHEP11(2011)012

[29] LeCompte T J and Martin S P 2011 Phys. Rev. D 84015004

[30] Allanach B and Gripaios B 2012 J. High Energy Phys. JHEP05(2012)062

[31] Graham P W, Kaplan D E, Rajendran S and Saraswat P 2012 J. High Energy Phys. JHEP07(2012)149

[32] Evans J A and Kats Y 2012 arXiv:1209.0764

[33] Brust C, Katz A and Sundrum R 2012 J. High Energy Phys. JHEP08(2012)059

[34] Dreiner H, Staub F, Vicente A and Porod W 2012 Phys. Rev. D 86035021

[35] Asano M, Rolsbiecki K and Sakurai K 2013 J. High Energy Phys. JHEP01(2013)128

[36] French S T (ATLAS Collaboration) 2010 AIP Conf. Proc. 1200354

[37] CMS Collaboration 20111193600 CMS-PAS-EXO-11-045

[38] Blanke M, Giudice G F, Paradisi P, Perez G and Zupan J 2013 J. High Energy Phys. JHEP06(2013)022

[39] Gedalia O, Kamenik J F, Ligeti Z and Perez G 2012 Phys. Lett. B 71455

[40] Nir Y and Seiberg N 1993 Phys. Lett. B 309337

[41] Galon I, Perez G and Shadmi Y 2013 J. High Energy Phys. JHEP09(2013)117

[42] Fitzpatrick A L, Perez G and Randall L 2008 Phys. Rev. Lett. 100171604

[43] Csaki C, Falkowski A and Weiler A 2009 Phys. Rev. D 80016001

[44] Mahbubani R, Papucci M, Perez G, Ruderman J T and Weiler A 2013 Phys. Rev. Lett. 110151804

[45] Bärnreuther P, Czakon M and Mitov A 2012 Phys. Rev. Lett. 109132001

[46] Czakon M and Mitov A 2012 J. High Energy Phys. JHEP12(2012)054

[47] Czakon M and Mitov A 2013 J. High Energy Phys. JHEP01(2013)080

[48] Czakon M, Fiedler P and Mitov A 2013 Phys. Rev. Lett. 110252004

[49] Mitov A 2013 CERN-TH colloquium (http://indico.cern.ch/conferenceDisplay.py?confId=245594)

[50] Czakon M, Fiedler P, Mitov A and Rojo J 2013 arXiv:1305.3892

[51] Martin A, Stirling W, Thorne R and Watt G 2009 Eur. Phys. J. C 63189

[52] Gao J et al 2013 arXiv:1302.6246

[53] Ball R D et al 2013 Nucl. Phys. B 867244

[54] Czakon M, Mangano M L, Mitov A and Rojo J 2013 J. High Energy Phys. JHEP07(2013)167

[55] Juste A et al 2013 arXiv: 1310.0799

[56] Aaltonen T et al (CDF Collaboration, D0 Collaboration) 2012 Phys. Rev. D 86092003

[57] Kuhn J H and Rodrigo G 1998 Phys. Rev. Lett. 8149

[58] Kuhn J H and Rodrigo G 1999 Phys. Rev. D 59054017 
[59] Bowen M, Ellis S and Rainwater D 2006 Phys. Rev. D 73014008

[60] Antunano O, Kuhn J H and Rodrigo G 2008 Phys. Rev. D 77014003

[61] Almeida L G, Sterman G F and Vogelsang W 2008 Phys. Rev. D 78014008

[62] Aaltonen T et al (CDF Collaboration) 2012 arXiv:1211.1003

[63] Abazov V M et al (D0 Collaboration) 2011 Phys. Rev. D 84112005

[64] Bernreuther W and Si Z-G 2012 Phys. Rev. D 86034026

[65] Aaltonen T A et al (CDF Collaboration) 2013 Phys. Rev. D 88072003

[66] D0 Collaboration 2013 Note Conf 6381

[67] Abazov V M et al (D0 Collaboration) 2013 arXiv:1308.6690

[68] CDF Collaboration 2013 Note 11035

[69] ATLAS Collaboration 2012 Technical Report ATLAS-CONF-2012-057 CERN, Geneva

[70] Chatrchyan S et al (CMS Collaboration) 2012 Phys. Lett. B 717129

[71] Chatrchyan S et al (CMS Collaboration) 2012 Phys. Lett. B 70928

[72] Aguilar-Saavedra J and Juste A 2012 Phys. Rev. Lett. 109211804
[73] Alvarez E and Leskow E C 2012 Phys. Rev. D 86114034

[74] Drobnak J, Kagan A L, Kamenik J F, Perez G and Zupan J 2012 arXiv: 1209.4872

[75] Drobnak J, Kamenik J F and Zupan J 2012 arXiv:1205.4721

[76] Kamenik J F, Shu J and Zupan J 2012 Eur. Phys. J. C 722102

[77] Falkowski A, Mangano M L, Martin A, Perez G and Winter J 2012 Phys. Rev. D 87034039

[78] Almeida L G, Lee S J, Perez G, Sung I and Virzi J 2009 Phys. Rev. D 79074012

[79] Choudhury D, Godbole R M, Rindani S D and Saha P 2011 Phys. Rev. D 84014023

[80] Krohn D, Liu T, Shelton J and Wang L-T 2011 Phys. Rev. D 84074034

[81] Berger E L, Cao Q-H, Chen C-R, Yu J-H and Zhang H 2012 Phys. Rev. Lett. 108072002

[82] Berger E L, Cao Q-H, Chen C-R and Zhang H 2012 arXiv: 1209.4899

[83] Cao J, Wu L and Yang J M 2011 Phys. Rev. D 83034024

[84] Falkowski A, Perez G and Schmaltz M 2011 arXiv:1110.3796

[85] Shaposhnikov M and Wetterich C 2010 Phys. Lett. B 683196

[86] Feldstein B, Hall L J and Watari T 2006 Phys. Rev. D 74095011

[87] Giudice G F, Perez G and Soreq Y 2012 arXiv:1207.4861 